\title{
Pacific
}

Journal of

Mathematics

\section{STRONG APPROXIMATE TRANSITIVITY, POLYNOMIAL GROWTH, AND SPREAD OUT RANDOM WALKS ON LOCALLY COMPACT GROUPS}

WOJCIECH JAWORSKI 


\title{
STRONG APPROXIMATE TRANSITIVITY, POLYNOMIAL GROWTH, AND SPREAD OUT RANDOM WALKS ON LOCALLY COMPACT GROUPS
}

\author{
WOJCIECH JAWORSKI
}

\begin{abstract}
We extend to continuous groups our recent results on strongly approximately transitive group actions. We are concerned with locally compact second countable groups and standard Borel $G$-spaces. A $G$-space $\mathcal{X}$ with a $\sigma$-finite quasiinvariant measure $\alpha$ is called strongly approximately transitive (SAT) if there exists a probability measure $\nu \ll \alpha$ such for every Borel set $A$ with $\alpha(A) \neq 0$ and every $\varepsilon>0$ one can find $g \in G$ with $\nu(g A)>1-\varepsilon$. Examples of SAT $G$-spaces include boundaries of spread out random walks on $G$. We prove that when $G$ is compactly generated and has polynomial growth then every standard SAT $G$-space $(\mathcal{X}, \alpha)$ is necessarily purely atomic; when $G$ is additionally connected, $(\mathcal{X}, \alpha)$ is a singleton. The Choquet-Deny theorem for spread out random walks on $G$ follows as a corollary. For a connected $G$ we establish the equivalence of the following conditions: (a) $G$ has polynomial growth; (b) every standard SAT G-space is a singleton; (c) every SAT homogeneous space of $G$ is a singleton; (d) every homogeneous space of $G$ admits a $\sigma$-finite invariant measure; (e) the Choquet-Deny theorem holds for every spread out probability measure on $G$; (f) the Choquet-Deny theorem holds for every absolutely continuous compactly supported probability measure on $G$.
\end{abstract}

\section{Introduction.}

The aim of this paper is to extend to continuous groups our recent results on strongly approximately transitive actions [9].

Let $G$ be a group and $\mathcal{X}$ a Borel $G$-space with a $\sigma$-finite quasiinvariant measure $\alpha$. We denote by $L^{1}(\mathcal{X}, \alpha)$ the space of complex measures absolutely continuous with respect to $\alpha$ and by $L_{1}^{1}(\mathcal{X}, \alpha) \subseteq L^{1}(\mathcal{X}, \alpha)$ the subspace of probability measures. For $g \in G$ and $\mu \in L^{1}(\mathcal{X}, \alpha)$ we write $g \mu$ for the measure $(g \mu)(A)=\mu\left(g^{-1} A\right) . B(G)$ denotes the space of bounded complex functions on $G$ equipped with the sup norm.

Consider the following properties that a probability measure $\rho \in L_{1}^{1}(\mathcal{X}, \alpha)$ might possess: 
(a) the convex hull $\operatorname{co}(G \rho)$ of the orbit $G \rho$ is dense in $L_{1}^{1}(\mathcal{X}, \alpha)$ with respect to the total variation norm;

(b) for every Borel set $A$ with $\alpha(A)>0$ and for every $\varepsilon>0$ there exists $g \in G$ such that $(g \rho)(A)=\rho\left(g^{-1} A\right)>1-\varepsilon$,

(c) the map $\mathcal{R}: L^{\infty}(\mathcal{X}, \alpha) \rightarrow B(G)$ given by $(\mathcal{R} f)(g)=\langle g \rho, f\rangle=$ $\int_{\mathcal{X}} f d(g \rho)$ is an isometry.

In [9, Proposition 2.2] we showed that conditions (a)-(c) are equivalent. A $G$-space $(\mathcal{X}, \alpha)$ admitting a probability measure $\rho \ll \alpha$ satisfying (a)-(c) is called strongly approximately transitive (SAT). A measure $\rho \in L_{1}^{1}(\mathcal{X}, \alpha)$ satisfying (a)-(c) is called a $S A T$ measure.

As shown in [9] every SAT action is ergodic. An ergodic action on a purely atomic measure space $(\mathcal{X}, \alpha)$ is trivially SAT. An ergodic $G$-space with a $\sigma$-finite invariant measure is SAT if and only if it is purely atomic. A contractive homogeneous space of a locally compact second countable group (e.g., the transitive action of the $a x+b$ group on $\mathbb{R}$ ) is a nonatomic SAT $G$-space. Boundaries of homogeneous random walks on $G$ provide further examples of nonatomic SAT actions. The boundary of an adapted random walk on a countable $G$ is either a singleton or a nonatomic SAT $G$-space. When $G$ is nonamenable the boundary is never a singleton; it also fails to be a singleton for certain adapted random walks on certain amenable $G$.

For finitely generated groups we linked the existence of nonatomic SAT actions to a growth condition on $G$. We showed that finitely generated groups of polynomial growth do not admit nonatomic SAT actions. This implies the Choquet-Deny theorem for such groups. Furthermore, for a finitely generated solvable $G$ we showed that $G$ has polynomial growth if and only if every SAT action is purely atomic. The purpose of the present work is to generalize these results to continuous locally compact second countable (lcsc) groups and sufficiently smooth Borel actions (on standard Borel spaces).

Using structure theory for lcsc compactly generated groups of polynomial growth [15] we show that such groups do not admit nonatomic SAT actions. Furthermore, for a connected lcsc group of polynomial growth every SAT Gspace is necessarily a singleton. As in the case of discrete (countable) groups treated in [9] we stress the interplay between the theory of SAT actions and the theory of boundaries of homogeneous random walks. A random walk of law $\mu$ is called spread out if $\mu$ is spread out, i.e., for some $n=1,2, \ldots$ the $n$-the convolution power $\mu^{n}$ is nonsingular with respect to the Haar measure. The boundary of a spread out random walk is always a SAT $G$-space (this can fail in the non spread out case). The fact that lcsc compactly generated groups do not admit nonatomic SAT actions implies the Choquet-Deny theorem for spread out random walks on such groups (see $[\mathbf{1}, \mathbf{7}, \mathbf{2 3}]$ for previous results in this direction). On the other hand, using the work of Azencott 
[2] and Raugi [21] on boundaries of random walks we show that for a connected lcsc $G$ the following conditions are equivalent: (a) $G$ has polynomial growth; (b) every standard SAT $G$-space is a singleton; (c) every SAT homogeneous space of $G$ is a singleton; (d) every homogeneous space of $G$ admits a $\sigma$-finite invariant measure; (e) the Choquet-Deny theorem holds for every spread out probability measure on $G$; (f) the Choquet-Deny theorem holds for every absolutely continuous compactly supported probability measure on $G$. The proof of this equivalence shows that if $G$ admits nonatomic SAT actions at all, it admits a SAT homogeneous space which is the boundary of a random walk defined by an absolutely continuous compactly supported probability measure.

\section{Preliminaries.}

2.1. Borel $G$-spaces. When $\mathcal{X}$ and $\mathcal{Y}$ are Borel $G$-spaces with $\sigma$-finite quasiinvariant measures $\alpha$ and $\beta$, we say that $(\mathcal{X}, \alpha)$ and $(\mathcal{Y}, \beta)$ are isomorphic if there exists an equivariant isomorphism of the $*$-algebra $L^{\infty}(\mathcal{X}, \alpha)$ onto $L^{\infty}(\mathcal{Y}, \beta)$. By the weak ${ }^{*}$ topology on $L^{\infty}(\mathcal{X}, \alpha)$ we mean the $\sigma\left(L^{\infty}, L^{1}\right)$ topology. We say that $(\mathcal{Y}, \beta)$ is a factor of $(\mathcal{X}, \alpha)$ if there exists an equivariant isomorphism of $L^{\infty}(\mathcal{Y}, \beta)$ onto a unital weakly* closed *-subalgebra of $L^{\infty}(\mathcal{X}, \alpha)$. We note that strong approximate transitivity is invariant with respect to isomorphisms and factors.

Our previous study of SAT [9] was primarily aimed at actions of discrete groups and our results did not rely on any particular assumptions about Borel structures involved. Our present study of SAT actions of lcsc groups relies on the following two routine technical assumptions about the $G$-spaces $(\mathcal{X}, \alpha)$ in question:

(i) $L^{1}(\mathcal{X}, \alpha)$ is separable;

(ii) for every $\varphi \in L^{1}(\mathcal{X}, \alpha)$ and $f \in L^{\infty}(\mathcal{X}, \alpha)$ the function $G \ni g \rightarrow$ $\langle\varphi, g f\rangle=\int f\left(g^{-1} x\right) \varphi(d x) \in \mathbb{C}$ is Borel.

As follows from Mackey's work [16], a $G$-space $(\mathcal{X}, \alpha)$ with the above two properties is always isomorphic to a $G$-space $(\mathcal{Y}, \beta)$ where $\mathcal{Y}$ is a standard Borel space on which $G$ acts so that the function $G \times \mathcal{Y} \ni(g, y) \rightarrow g y \in \mathcal{Y}$ is Borel. Such $G$-spaces (with $G$ lcsc) will be referred to as standard. To simplify the exposition our results will be formulated and proven for standard $G$-spaces.

Remark 2.1. Suppose that $G$ acts on $L^{\infty}(\mathcal{X}, \alpha)$ by $*$-algebra automorphisms and we do not assume that this action is induced by an action on $\mathcal{X}$. The dual action on $L^{1}(\mathcal{X}, \alpha)$ is then defined by formula $\langle g \varphi, f\rangle=$ $\left\langle\varphi, g^{-1} f\right\rangle, \varphi \in L^{1}(\mathcal{X}, \alpha), f \in L^{\infty}(\mathcal{X}, \alpha)$ and it is obvious that strong approximate transitivity is meaningful in this setting. Due to Mackey [16], 
under the regularity conditions (i), (ii) there is no loss of generality in discussing standard $G$-spaces only. However, certain portion of our argument in the sequel is most conveniently carried out in the setting of actions on $L^{\infty}$-spaces. We shall occasionally work in this setting without additional comments.

The following lemma will be very important.

Lemma 2.2. Let $G$ be a lcsc group acting on $L^{\infty}(\mathcal{X}, \alpha)$ by *-algebra automorphism. Assume that $L^{1}(\mathcal{X}, \alpha)$ is separable for every $\varphi \in L^{1}(\mathcal{X}, \alpha)$ and $f \in L^{\infty}(\mathcal{X}, \alpha)$ the function $G \ni g \rightarrow\langle\varphi, g f\rangle \in \mathbb{C}$ is Borel. Then for every $\varphi \in L^{1}(\mathcal{X}, \alpha)$ the function $G \ni g \rightarrow g \varphi \in L^{1}(\mathcal{X}, \alpha)$ is continuous (in $L^{1}-$ norm $)$.

Proof. Let $I$ denote the group of isometric isomorphisms of $L^{1}(\mathcal{X})=L^{1}(\mathcal{X}, \alpha)$. Since $L^{1}(\mathcal{X})$ is a separable Banach space, $I$ equipped with the strong operator topology is a separable metrizable group and the induced Borel structure is standard [26, Lemma 1.1]. A routine argument using separability of $L^{1}(\mathcal{X})$ and the uniqueness theorem for analytic Borel structures [18, Corollary 2.10] shows that the Borel structure on $I$ is the smallest such that for every $\varphi \in L^{1}(\mathcal{X})$ and $f \in L^{\infty}(\mathcal{X})$ the function $I \ni T \rightarrow\langle T \varphi, f\rangle \in \mathbb{C}$ is Borel. Now, the dual action of $G$ on $L^{1}(\mathcal{X})$ means that we have a homomorphism of $G$ into $I$. Our assumption guaranties that this homomorphism is a Borel map. But a Borel homomorphism of a lcsc group into a second countable group is necessarily continuous [27, Theorem B3; p. 198].

2.2. Random walks. Consider the right random walk of law $\mu$ on a lcsc group $G$. Let $G^{\infty}=\prod_{n=0}^{\infty} G$ be the space of paths with the Borel structure given by the product $\sigma$-algebra and let $\rho$ denote the Markov measure of the random walk started from identity $e$ of $G$. Equip $G^{\infty}$ with the $G$-action $g\left\{\omega_{n}\right\}_{n=0}^{\infty}=\left\{g \omega_{n}\right\}_{n=0}^{\infty}$. Since $G$ is lcsc, $G^{\infty}$ becomes a standard $G$-space. Furthermore, if $\alpha_{0}$ is a probability measure on $G$ equivalent to the Haar measure then the measure $\alpha=\alpha_{0} * \rho$ (the Markov measure with starting measure $\alpha_{0}$ ) is a quasiinvariant measure on $G^{\infty}$. Denote by $\mathcal{X}$ the $G$-space $G^{\infty}$ with the Borel structure given by the invariant (stationary) $\sigma$-algebra of the random walk. The triple $(\mathcal{X}, \alpha, \rho)$ will be called the boundary or $\mu$-boundary of the random walk (of law $\mu$ ). The $\mu$-boundary is a $G$-space but fails to be standard unless $G=\{e\}$ (the invariant $\sigma$-algebra does not separate points). However, our regularity conditions (i) and (ii) introduced in Section 2.1 are satisfied and the $\mu$-boundary can be always realized as a standard $G$-space. 
A bounded Borel function $h \in B(G)$ is called $\mu$-harmonic if

$$
h(g)=\int_{G} h\left(g g^{\prime}\right) \mu\left(d g^{\prime}\right), \quad g \in G .
$$

By the basic theory of Markov chains there exists an isometric isomorphism $\Phi$ between equivalence classes of bounded Borel functions on $\mathcal{X}$ and $\mu$-harmonic functions [17, Proposition V.2.4; p. 175], [20, Proposition 3.2; p. 82]. Two bounded Borel functions $f_{1}, f_{2}$ on $\mathcal{X}$ are declared equivalent if $\left\langle g \rho,\left|f_{1}-f_{2}\right|\right\rangle=$ 0 for all $g \in G$. $\Phi$ is given by

$$
h(g)=(\Phi f)(g)=\langle g \rho, f\rangle=\int_{\mathcal{X}} f(g x) \rho(d x) .
$$

The law $\mu$ is called spread out if for some $n=1,2, \ldots$ the $n$-the convolution power $\mu^{n}$ is nonsingular with respect to the Haar measure. It can be shown that for spread out $\mu$ every $\mu$-harmonic function is continuous [2, Proposition I.6; p. 23]. This immediately implies that the measure $\rho$ is absolutely continuous with respect to $\alpha=\alpha_{0} * \rho$ (on the invariant $\sigma$ algebra). Therefore Eq. (2.1) define an equivariant isometric isomorphism of $L^{\infty}(\mathcal{X}, \alpha)$ onto the space of $\mu$-harmonic functions. In consequence, $(\mathcal{X}, \alpha)$ is a SAT $G$-space and $\rho$ is a SAT measure.

The random walk is called adapted if $\mu(H)<1$ for every proper closed subgroup of $G$. The boundary measure $\rho$ is referred to as trivial if $\rho(A) \epsilon$ $\{0,1\}$ for every Borel subset of $\mathcal{X}$. We note that a SAT measure on a (general) $G$-space is trivial if and only if the $G$-space is purely atomic.

Lemma 2.3. Let $\mu$ be a spread out probability measure on a lcsc group $G$ and $(\mathcal{X}, \alpha, \rho)$ the associated $\mu$-boundary. Then

(a) the action of $G$ on $(\mathcal{X}, \alpha)$ is SAT and $\rho$ is a SAT measure,

(b) $\rho$ is trivial if and only if every $\mu$-harmonic function is constant on the left cosets of the smallest closed subgroup $H \subseteq G$ such that $\mu(H)=1$,

(c) when $\mu$ is adapted, $\rho$ is trivial if and only if every $\mu$-harmonic function is constant.

Remark 2.4. When $\mu$ is arbitrary, Formula (2.1) induces an equivariant weak* continuous isometry of $L^{\infty}(\mathcal{X}, \alpha)$ into $L^{\infty}(G, \lambda)(\lambda=$ the Haar measure). $L^{\infty}(\mathcal{X}, \alpha)$ is then isomorphic to the space of equivalence classes ( $\bmod$ $\lambda$ ) of $\mu$-harmonic functions. In the trivial case that $\mu=\delta_{e}$ the $\mu$-boundary is isomorphic to $G$. Thus for continuous $G$ the $\delta_{e}$-boundary is not SAT. Motivated by this situation one can define a weak version of SAT by requiring that there exists an equivariant weak* continuous isometry of $L^{\infty}(\mathcal{X})$ into $L^{\infty}(G)$. For countable $G$ this property is equivalent to SAT but not 
so for continuous $G$ (every homogeneous space admits an equivariant weak* continuous isometry into $\left.L^{\infty}(G)\right)$. It is known that for abelian lcsc $G$ every equivariant weak* continuous isometry of $L^{\infty}(\mathcal{X})$ into $L^{\infty}(G)$ (with $\mathcal{X}$ standard) is necessarily multiplicative and such an isometry exists if and only if $G$ is essentially transitive on $\mathcal{X}[\mathbf{1 0}]$. This result implies the Choquet-Deny theorem for arbitrary $\mu$.

\section{Strong approximate transitivity and polynomial growth.}

Unless explicitly stated, all $G$-spaces in the sequel are assumed standard and all groups lcsc.

\subsection{Compact and cocompact subgroups.}

Proposition 3.1. Let $N$ be a compact normal subgroup of $G$. If every SAT $(G / N)$-space is purely atomic then every SAT $G$-space purely atomic.

Proof. Let $\mathcal{X} / N$ be the orbit space and $p: \mathcal{X} \rightarrow \mathcal{X} / N$ the quotient map. Equip $\mathcal{X} / N$ with the quotient Borel structure. As $N$ is compact this Borel structure is standard (combine [4, Theorem 2.9] with [24, Theorem 3.21]). Since $N \triangleleft G$ we can define a $G$-action on $\mathcal{X} / N$ by $g p(x)=p(g x)$. For this action the map $G \times(\mathcal{X} / N) \ni(g, x) \rightarrow g x$ is Borel. (This follows immediately from the fact that the product Borel structure on $G \times(\mathcal{X} / N)$ coincides with the quotient Borel structure induced by the map $G \times \mathcal{X} \ni(g, x) \rightarrow(g, p(x))$; this in turn is a consequence of the uniqueness theorem for analytic Borel structures [18, Corollary 2.10].) The $G$-space $(\mathcal{X} / N, p \alpha)$ is a factor of the $G$ space $(\mathcal{X}, \alpha)$. Therefore it is SAT and, consequently, due to our assumption, $p \alpha$ is carried on a countable set $C \subseteq \mathcal{X} / N$. Since SAT actions are ergodic, there exists a countable set $\Gamma \subseteq G$ and $x \in \mathcal{X}$ such that $C=\Gamma p(x)$. Hence, $\alpha$ is carried on $\Gamma N x$ which is a countable union of disjoint orbits of $N$. As $N$ is compact its Haar measure $\lambda_{N}$ is finite. We can assume that $\alpha$ is also finite. Then $\lambda_{N} * \alpha$ is a finite $N$-invariant measure equivalent to $\alpha$. As $\lambda_{N} * \alpha$ is carried on a countable set of translates of a single orbit of $N$ it is clear that there exists a $\sigma$-finite $G$-invariant measure equivalent to $\alpha$. Since SAT actions preserving a $\sigma$-finite invariant measure are necessarily purely atomic $[9$, Proposition 2.6], the proof is complete.

Lemma 3.2. Let $(\mathcal{X}, \alpha)$ be a $G$-space and $K \subseteq L_{1}^{1}(\mathcal{X}, \alpha)$ a compact subset such that for every Borel set $A \subseteq \mathcal{X}$ with $\alpha(A) \neq 0$ and every $\varepsilon>0$ there exists $g \in G$ and $\nu \in K$ such that $(g \nu)>1-\varepsilon$. Then there exists a $G$ invariant Borel set $\mathcal{X}_{0} \subseteq \mathcal{X}$ and $\nu_{0} \in K$ such that $\nu_{0}\left(\mathcal{X}_{0}\right)=1$ and $\nu_{0}$ is a 
$S A T$ measure on $\left(\mathcal{X}_{0}, \alpha_{0}\right)$ where $\alpha_{0}$ denotes the restriction of $\alpha$ to $\mathcal{X}_{0}$.

Proof. Step I: Let $\Omega \subseteq \mathcal{X}$ be a $G$-invariant Borel set with $\alpha(\Omega) \neq 0$ and $M$ a compact subset of $K$ such that for every Borel $A \subseteq \Omega$ with $\alpha(A) \neq 0$ and every $\varepsilon>0$ there exists $g \in G$ and $\nu \in M$ such that $(g \nu)>1-\varepsilon$. We prove that for every $r>0$ there exists a nonempty compact subset $M_{*} \subseteq M$ of diameter at most $r$ and a $G$-invariant Borel set $\Omega_{*} \subseteq \Omega$ such that $\nu\left(\Omega_{*}\right)=1$ for every $\nu \in M_{*}$ and that for every Borel set $A \subseteq \Omega_{*}$ with $\alpha(A) \neq 0$ and every $\varepsilon>0$ there exists $g \in G$ and $\nu \in M_{*}$ such that $(g \nu)(A)>1-\varepsilon$.

Let $\bar{B}(\nu, r) \subseteq L^{1}(\mathcal{X})$ denote the closed ball with center $\nu$ and radius $r$. Due to the compactness of $M$ we can find a sequence $\nu_{1}, \ldots, \nu_{k} \in M$ such that $M \subseteq \bigcup_{i=1}^{k} \bar{B}\left(\nu_{i}, r / 2\right)$. Set $M_{i}=\bar{B}\left(\nu_{i}, r / 2\right) \cap M$ and let $\mathcal{F}_{i}$ denote the collection of Borel sets $A \subseteq \Omega$ such that $\alpha(A) \neq 0$ and for every $\varepsilon>0$ and every Borel $B \subseteq A$ with $\alpha(B) \neq 0$ there exists $g \in G$ and $\nu \in M_{i}$ with $(g \nu)(B)>1-\varepsilon$. We claim that $\mathcal{F}_{i} \neq \varnothing$ for at least one $i \in\{1,2, \ldots, k\}$. Indeed, it is easy to see that if $\mathcal{F}_{i} \neq \varnothing$ for all $i \in\{1,2, \ldots, k\}$, then there would exist a sequence $\varepsilon_{1}, \ldots, \varepsilon_{k}>0$ and a sequence of Borel sets $\Omega \supseteq B_{1} \supseteq$ $B_{2} \supseteq \cdots \supseteq B_{k}$ such that for every $i=1,2, \ldots, k \alpha\left(B_{i}\right) \neq 0$ and $(g \nu)\left(B_{i}\right) \leq$ $1-\varepsilon_{i}$ for all $g \in G$ and all $\nu \in M_{i}$. Set $\varepsilon=\min \left\{\varepsilon_{1}, \ldots, \varepsilon_{k}\right\}$. According to our assumption there exists $g \in G$ and $\nu \in M$ with $(g \nu)\left(B_{k}\right)>1-\varepsilon$. Since $\nu$ belongs to some $M_{i}$ we obtain $(g \nu)\left(B_{i}\right) \geq(g \nu)\left(B_{k}\right)>1-\varepsilon \geq 1-\varepsilon_{i}$, in contradiction with the definition of the sequences $B_{1}, \ldots, B_{k}$ and $\varepsilon_{1}, \ldots, \varepsilon_{k}$.

Let $\mathcal{F}_{i} \neq \varnothing$ and let $\mathcal{E}$ be the corresponding family of projections of $L^{\infty}(\mathcal{X})$, i.e., $\varphi \in \mathcal{E}$ if and only if $\varphi=\chi_{A}(\bmod \alpha)$ and $A \in \mathcal{F}_{i}$. It is clear that $\mathcal{E}$ is a $G$-invariant family. Therefore $\psi=\vee \mathcal{E}$ is a $G$-invariant projection. As $\mathcal{X}$ is a standard $G$-space, $\psi=\chi_{\Omega_{*}}(\bmod \alpha)$ for a $G$-invariant Borel set $\Omega_{*} \subseteq \Omega$ [27, Lemma 2.2 .16 ; p. 21]. It is easy to see that $\Omega_{*} \subseteq \mathcal{F}_{i}$. The $G$-invariance of $\Omega_{*}$ implies that there exists a sequence $\nu_{n}$ in $M_{i}$ such that $\nu_{n}\left(\Omega_{*}\right)>1-\frac{1}{n}$. By the compactness of $M_{i}$ we may assume that $\nu_{n}$ converges to some $\nu \in M_{i}$. Clearly, $\nu\left(\Omega_{*}\right)=1$. Set $M_{*}=\left\{\nu \in M_{i} ; \nu\left(\Omega_{*}\right)=1\right\}$. This is a nonempty compact subset of $L_{1}^{1}(\mathcal{X})$ of diameter at most $r$. Let us see that for every Borel $A \subseteq \Omega_{*}$ with $\alpha(A) \neq 0$ and every $\varepsilon>0$ there exists $g \in G$ and $\nu \in M_{*}$ such that $\nu(A)>1-\varepsilon$. Indeed, as $\Omega_{*} \subseteq \mathcal{F}_{i}$, from the definition of $\mathcal{F}_{i}$ there exists a sequence $\nu_{n}$ in $M_{i}$ and a sequence $g_{n}$ in $G$ such that $\left(g_{n} \nu_{n}\right)(A)>1-\frac{1}{n}$. By the compactness we may assume that $\nu_{n}$ converges to some $\nu \in M_{i}$. It is clear that $\nu\left(\Omega_{*}\right)=1$, so that $\nu \in M_{*}$. Furthermore, $\left(g_{n} \nu\right)(A) \geq\left(g_{n} \nu_{n}\right)(A)-\left\|\nu_{n}-\nu\right\| \rightarrow 1$. Our claim is proven.

Step II: Using Step I we can inductively produce a decreasing sequence of $G$-invariant Borel sets $\Omega_{n}$ and a decreasing sequence $M_{n}$ of nonempty compact subset of $K$ such that $M_{n}$ has diameter at most $1 / n$, that $\nu\left(\Omega_{n}\right)=1$ for all $\nu \in M_{n}$, and that for every Borel $A \subseteq \Omega_{n}$ with $\alpha(A) \neq 0$ and every 
$\varepsilon>0$ there exists $g \in G$ and $\nu \in M_{n}$ such that $(g \nu)(A)>1-\varepsilon$. The finite intersection property of the sequence $M_{n}$ implies that $\bigcap_{n=1}^{\infty} M_{n}$ contains precisely one element $\nu_{0}$. Let $\mathcal{X}_{0}=\bigcap_{n=1}^{\infty} \Omega_{n}$. Clearly, $\mathcal{X}_{0}$ is $G$-invariant and $\nu_{0}\left(\mathcal{X}_{0}\right)=1$. Our proof will be complete if we show that $\nu_{0}$ is a SAT measure on $\mathcal{X}_{0}$. Let $A \subseteq \mathcal{X}_{0}$ be a Borel set with $\alpha(A) \neq 0$. Since $A \subseteq \Omega_{n}$ for every $n$, there exist $g_{n} \in G$ and $\nu_{n} \in M_{n}$ such that $\left(g_{n} \nu_{n}\right)(A)>1-\frac{1}{n}$. But $\left\|\nu_{0}-\nu_{n}\right\| \leq 1 / n$. Therefore $\left(g_{n} \nu_{0}\right)(A) \geq 1-\frac{2}{n}$. So $\nu_{0}$ is a SAT measure on the $G$-space $\left(\mathcal{X}_{0}, \alpha_{0}\right)$.

Proposition 3.3. Let $H$ be a closed cocompact subgroup of $G$. If every SAT $H$-space is purely atomic then every SAT G-space is purely atomic. Furthermore, if $(\mathcal{X}, \alpha)$ is a SAT $G$-space and $\rho$ a SAT measure, then there exists an $H$-invariant Borel set $\mathcal{X}_{0} \subseteq \mathcal{X}$ and $g_{0} \in G$ such that $\left(g_{0} \rho\right)\left(\mathcal{X}_{0}\right)=1$ and $g_{0} \rho$ is a SAT measure for the action of $H$ on $\left(\mathcal{X}_{0}, \alpha_{0}\right)$ where $\alpha_{0}$ is the restriction of $\alpha$ to $\mathcal{X}_{0}$.

Proof Since $H$ is cocompact, there exists a compact set $F \subseteq G$ such that $H F \stackrel{=}{=}$. By Lemma $2.2 K=F \rho$ is a compact subset of $L_{1}^{1}(\mathcal{X})$ and the assumptions of Lemma 3.2 satisfied with respect to the action of $H$ on $(\mathcal{X}, \alpha)$.

3.2. Compactly generated groups of polynomial growth. The following lemma will be eventually applied in an inductive argument when $G$ is a Lie group and $H$ a normal Lie subgroup.

Lemma 3.4. Let $H$ be a (not necessarily closed) normal subgroup of $G$. Suppose $H$ is equipped with a connected locally connected group topology which contains the relative topology and for every neighbourhood $U$ of $e$ in $G$ there exists a neighbourhood $V$ of $e$ in $H$ such that $g_{V g^{-1}} \subseteq U$ for all $g \in G$. Let $(\mathcal{X}, \alpha)$ be a SAT $G$-space. Then $h f=f$ for every $f \in L^{\infty}(\mathcal{X}, \alpha)$ and $h \in \bar{H}$.

Proof. Let $\rho$ be a SAT measure on $\mathcal{X}$. Set $a(g)=\|g \rho-\rho\|$ and $b(h)=$ $\sup _{g \in G} a\left(g h g^{-1}\right), h \in G$. Let $U$ be a compact neighbourhood of $e$ in $G$ and $V$ a connected neighbourhood of $e$ in $H$ such that $g V g^{-1} \subseteq U$ for all $g \in G$. We claim that the function $b(h)$ is continuous on $V$. To see this suppose the contrary: $b$ is discontinuous at some $h \in V$. Then there exists $\varepsilon>0$ and a net $h_{\beta}$ in $V$ converging to $h$ and such that $\left|b\left(h_{\beta}\right)-b(h)\right| \geq \varepsilon$ for all $\beta$. Using the definition of $b$, for every $\beta$ we can then find $g_{\beta} \in G$ such that $\left|a\left(g_{\beta} h_{\beta} g_{\beta}^{-1}\right)-a\left(g_{\beta} h g_{\beta}^{-1}\right)\right| \geq \varepsilon / 2$ (consider the cases $b\left(h_{\beta}\right)-b(h) \geq \varepsilon$ and $\left.b(h)-b\left(h_{\beta}\right) \geq \varepsilon\right)$. Since $U$ is compact and $g V g^{-1} \subseteq U$ for all $g \in G$, we can assume that the nets $g_{\beta} h_{\beta} g_{\beta}^{-1}$ and $g_{\beta} h g_{\beta}^{-1}$ converge in $U: g_{\beta} h_{\beta} g_{\beta}^{-1} \rightarrow$ 
$x, g_{\beta} h g_{\beta}^{-1} \rightarrow y$. Since $a$ is a continuous function we obtain that $x \neq y$. However, consider the net $g_{\beta} h_{\beta} h^{-1} g_{\beta}^{-1}$. Clearly, $g_{\beta} h_{\beta} h^{-1} g_{\beta}^{-1} \rightarrow x y^{-1}$. Let $U^{\prime}$ be any neighbourhood of $e$ in $G$ and $V^{\prime}$ a neighbourhood of $e$ in $H$ with $g V^{\prime} g^{-1} \subseteq U^{\prime}$ for all $g \in G$. Since $h_{\beta} h^{-1}$ converges to $e$ in $H$, we conclude that $g_{\beta} h_{\beta} h^{-1} g_{\beta}^{-1}$ converges in $G$ to $e$. Hence, $x=y$, a contradiction.

By [9, Proposition 3.1] $b(g) \in\{0,2\}$ for every $g \in G$. As $b$ is continuous on $V, b(e)=0$, and $V$ is connected, we conclude that $b(h)=0$ for all $h \in V$. Thus, $h \rho=\rho$ for all $h \in V$. But the set $\{h \in H ; h \rho=\rho\}$ is a subgroup of $H$ containing $V$. As $H$ is connected, it is generated by $V$. Therefore $h \rho=\rho$ for $h \in H$. Since the function $G \ni g \rightarrow g \rho$ is continuous we obtain $g \rho=\rho$ for $g \in \bar{H}$. Using the fact that $\bar{H}$ is normal and $\operatorname{co}(G \rho)$ is dense in $L_{1}^{1}(\mathcal{X})$, we conclude that $g \mu=\mu$ for $g \in \bar{H}$ and $\mu \in L_{1}^{1}(\mathcal{X})$. This, of course, implies that $g f=f$ for $g \in \bar{H}$ and $f \in L^{\infty}(\mathcal{X})$.

Let $\mathfrak{h}$ a (real) Lie algebra and $G$ a group acting on $\mathfrak{h}$ by automorphisms. Following [15] we say that $\mathfrak{h}$ is of type $R_{G}$ if for every $g \in G$ the eigenvalues of the corresponding automorphism are of absolute value 1 . When $G$ is locally compact and $H$ a closed normal Lie subgroup with Lie algebra $\mathfrak{h}$, this definition refers to the canonical adjoint action of $G$ on $\mathfrak{h}$. When $G$ is a connected Lie group with Lie algebra $\mathfrak{g}$, then $\mathfrak{g}$ is of type $R_{G}$ if and only if for every $X \in \mathfrak{g}$ the eigenvalues of $\operatorname{ad} X$ are imaginary [13, Proposition 1.3']. In this case the term type $R$ is commonly used instead of type $R_{G}$.

Lemma 3.5. Let $G$ be a Lie group and $H$ a closed normal Lie subgroup with $\operatorname{dim} H<\operatorname{dim} G$. If the Lie algebra of $G$ is of type $R_{G}$ then the Lie algebra of $G / H$ is of type $R_{G / H}$.

Proof. Let $\mathfrak{g}$ and $\mathfrak{h}$ be the Lie algebras of $G$ and $H$, respectively. Let $\pi$ : $G \rightarrow G / H$ and $\pi_{*}: \mathfrak{g} \rightarrow \mathfrak{g} / \mathfrak{h}$ denote the canonical homomorphisms. Let $b_{1}, \ldots, b_{k}, b_{k+1}, \ldots, b_{n}$ be a basis in $\mathfrak{g}$ such that $b_{k+1}, \ldots, b_{n}$ is a basis in $\mathfrak{h}$. Then $\pi_{*}\left(b_{1}\right), \ldots, \pi_{*}\left(b_{k}\right)$ is a basis in $\mathfrak{g} / \mathfrak{h}$. Let $A(g)$ be the matrix representing $\operatorname{Ad}(g)$ with respect to $b_{1}, \ldots, b_{n}$ and $\widetilde{A}(g)$ the matrix representing $\operatorname{Ad}(\pi(g))$ with respect to $\pi_{*}\left(b_{1}\right), \ldots, \pi_{*}\left(b_{k}\right)$. Since $\pi_{*} \circ \operatorname{Ad}(g)=\operatorname{Ad}(\pi(g)) \circ \pi_{*}$ it follows that $\widetilde{A}(g)_{i j}=A(g)_{i j}$ for $i, j=1, \ldots, k$. But $A(g)_{i j}=0$ for $i=1, \ldots, k ; j=$ $k+1, \ldots, n$ because $\mathfrak{h}$ is invariant under $\operatorname{Ad}(G)$. Thus every eigenvalue of $\operatorname{Ad}(\pi(g))$ is an eigenvalue of $\operatorname{Ad}(g)$.

Lemma 3.6. Let $G$ be a solvable Lie group and $G_{0}$ the identity component. Assume that $G / G_{0}$ is nilpotent and the Lie algebra of $G$ is of type. $R_{G}$. Then every SAT G-space is purely atomic.

Proof. We proceed by induction in the dimension of the Lie algebra $\mathfrak{g}$ of $G$. When $\operatorname{dim} \mathfrak{g}=1$, then obviously $\operatorname{Ad}(G) \subseteq\{ \pm 1\}$. Let $U$ be a neighbourhood 
of $e$ and $W=-W \subseteq \mathfrak{g}$ a neighbourhood of 0 such that $V=\exp (W) \subseteq U$. Clearly, $g V g^{-1}=\exp (\operatorname{Ad}(g) W)=\exp (W) \subseteq U$. By Lemma 3.4 $H=G_{0}=$ $\exp (\mathfrak{g})$ acts trivially on $L^{\infty}(\mathcal{X})$. Therefore we can define a SAT action of $G / H$ on $L^{\infty}(\mathcal{X})$ by $(g H) f=g f$. Since $G / H$ is countable nilpotent, $(\mathcal{X}, \alpha)$ is purely atomic [9, Theorem 3.9].

Suppose now that the lemma is already proven for dimensions lower than $n+1$ and let $\operatorname{dim} \mathfrak{g}=n+1$. Let $\mathfrak{g}_{\mathbb{C}}$ denote a complexification of $\mathfrak{g}$. Then $\operatorname{Ad}(G)$ can be considered as a solvable subgroup of $G L\left(\mathfrak{g}_{\mathbb{C}}\right)$. According to Kolchin-Malcev theorem [14, Theorem 21.1.5; p. 152] there exists a subgroup $S \subseteq \operatorname{Ad}(G)$ of finite index and a basis $b_{1}, \ldots, b_{n+1}$ in $\mathfrak{g}_{\mathbb{C}}$ such that every element $s \in S$ is represented by an upper triangular matrix with respect to this basis. The inverse image $G_{1}=\operatorname{Ad}^{-1}(S) \subseteq G$ is a subgroup of finite index. Note that we can assume that $G_{1}$ is closed. Hence, $G_{1}$ is an open Lie subgroup and $\mathfrak{g}$ is its Lie algebra. Note also that $G_{0}=G_{10}$. Consequently $G_{1} / G_{10}$ is nilpotent.

Let $\rho$ be a SAT measure on $\mathcal{X}$. By Proposition 3.3 there exists a $G_{1}$ invariant Borel set $\mathcal{X}_{1} \subseteq \mathcal{X}$ and $g_{1} \in G$ such that $g_{1} \rho$ is a SAT measure for the action of $G_{1}$ on $\mathcal{X}_{1}$. It suffices to show that $g_{1} \rho$ is a point measure.

Clearly, the elements of $\operatorname{Ad}\left(G_{1}\right)$ have a common eigenvector $v \in \mathfrak{g}_{\mathbb{C}}$. The complex conjugate $\bar{v}$ is also a common eigenvector of $\operatorname{Ad}\left(G_{1}\right)$. It follows that the (real) subspace $\mathfrak{h}$ of $\mathfrak{g}$ spanned by $w_{1}=\operatorname{Re} v$ and $w_{2}=\operatorname{Im} v$ is a subspace invariant under $\operatorname{Ad}\left(G_{1}\right)$, of dimension 1 or 2 . Since $\operatorname{Ad}(g)$ has eigenvalues of absolute value 1 , it can be seen that the restriction of $\operatorname{Ad}\left(G_{1}\right)$ to $\mathfrak{h}$ is contained in a compact subgroup $K$ of $G L(\mathfrak{h})$. Since $\mathfrak{h}$ is invariant under $\operatorname{Ad}\left(G_{1}\right)$ it is a subalgebra of $\mathfrak{g}$ and the connected Lie subgroup $H$ defined by $\mathfrak{h}$ is normal in $G_{1}$. Let $U$ be a neighbourhood of $e$ in $G_{1}$ and $W$ a neighbourhood of 0 in $\mathfrak{h}$ such that $\exp (W) \subseteq U$. Since $K$ is compact we can find a neighbourhood $0 \in W^{\prime} \subseteq W$ such that $K W^{\prime} \subseteq W$. Put $V=\exp \left(W^{\prime}\right)$. Then for every $g \in G_{1}$ we obtain $g V g^{-1} \subseteq \exp \left(\operatorname{Ad}(g) W^{\prime}\right) \subseteq \exp \left(K W^{\prime}\right) \subseteq$ $\exp (W) \subseteq U$. Thus the assumptions of Lemma 3.4 are satisfied and we conclude that $\bar{H}$ acts trivially on $L^{\infty}\left(\mathcal{X}_{1}\right)$. We can then consider $L^{\infty}\left(\mathcal{X}_{1}\right)$ as a SAT $\left(G_{1} / \bar{H}\right)$-space. If $\bar{H}$ is open in $G_{1}$ then $\bar{H}=G_{10}$ and $G_{1} / \bar{H}$ is nilpotent. From [9, Theorem 3.9] we obtain that $g_{1} \rho$ is a point measure. If $\bar{H}$ is not open then $G_{1} / \bar{H}$ is a solvable Lie group with Lie algebra of type $R_{G_{1} / \bar{H}}$ and dimension lower than $n+1$. Since $\left(G_{1} / \bar{H}\right)_{0}=G_{10} / \bar{H},\left(G_{1} / \bar{H}\right) /\left(G_{1} / \bar{H}\right)_{0}$ is nilpotent. By induction, $L^{\infty}\left(\mathcal{X}_{1}\right)$ is purely atomic and $g_{1} \rho$ is a point measure. 
growth. Then $G$ does not admit nonatomic SAT actions.

Proof. First consider the case that $G$ is a Lie group. According to [15, Corollary to Theorem 2] there exists a normal series $C \leq S \leq N \leq G$ such that $C$ and $G / N$ are compact, $S / C$ is connected with Lie algebra of type $R_{G / C}$, and $N / S$ is discrete nilpotent. Furthermore, $S / C$ can be assumed solvable. Note that $S / C=(N / C)_{0}$. It follows that $N / C$ is solvable with Lie algebra of type $R_{N / C}$ and $(N / C) /(N / C)_{0}$ is nilpotent. Thus from Lemma 3.6 we conclude that $N / C$ does not admit nonatomic SAT actions. Since $C$ is compact, by Proposition $3.1 N$ does not admit nonatomic SAT actions. But $G / N$ is compact. Thus from Proposition $3.3 G$ does not admit nonatomic SAT actions.

Now let $G$ be an arbitrary lcsc compactly generated group of polynomial growth. By [15, Theorem 2] there exists a compact normal subgroup $K$ such that $G / K$ is a Lie group (of polynomial growth). Another application of Proposition 3.1 finishes the proof.

Corollary 3.8. Let $\mu$ be a spread out probability measure on a lcsc compactly generated group of polynomial growth. Then every $\mu$-harmonic function is constant on the cosets of the smallest closed subgroup $H \subseteq G$ such that $\mu(H)=1$. If $\mu$ is adapted then every $\mu$-harmonic function is constant.

Remark 3.9. Theorem 3.7 remains true whenever $G$ is a lcsc (not necessarily compactly generated) group containing a closed nilpotent subgroup $H$ such that $G / H$ is compact. By Proposition 3.3 to prove this result it suffices to consider the case that $G$ is nilpotent. In [9] we gave a proof valid for countable $G$ [9, Theorem 3.9]. This proof after suitable technical modifications works also in the lcsc case.

Gromov's theorem [6] states that every finitely generated group of polynomial growth contains a nilpotent subgroup of finite index. Therefore in the countable case Theorem 3.7 is a direct consequence of Proposition 3.3 and the fact that nilpotent groups do not admit nonatomic SAT actions. In the continuous case an analog of Gromov's theorem stating that $G$ contains a nilpotent subgroup $H$ with $G / H$ compact is false and we had to rely on a more complicated structure theory $[\mathbf{1 5}]$.

3.3. Connected groups of polynomial growth. It can be easily shown that a probability measure $\mu$ on $G$ is spread out if and only if there exists $n$ such that $\mu^{n}$ dominates a positive multiple of the Haar measure on a nonempty open subset of $G\left[2\right.$, p. 21]. Hence, $\operatorname{supp} \mu^{n}$ has nonempty interior and the smallest closed subgroup $H \subseteq G$ with $\mu(H)=1$ is necessarily open. Consequently, when $G$ is connected, a spread out $\mu$ is automatically adapted 
and Corollary 3.8 says that the $\mu$-boundary of every spread out random walk on a connected lcsc group of polynomial growth is a singleton. This result, however, is also contained in the following stronger consequence of Theorem 3.7 and Lemma 2.2 .

Corollary 3.10. If $G$ is a connected lcsc group of polynomial growth then every $\mathrm{SAT} G$-space $(\mathcal{X}, \alpha)$ is a singleton.

Proof. We already know that $\alpha$ is purely atomic. Hence, if $\rho$ is a SAT measure it is carried on a single atom of $\alpha$. Consequently, $\|g \rho-\rho\|=\{0,2\}$ for every $g \in G$. But by Lemma 2.2 the function $g \rightarrow\|g \rho-\rho\|$ is continuous. Therefore $g \rho=\rho$ for all $g \in G$. Since $\rho$ is SAT we have that $g f=f$ for $f \in L^{\infty}(\mathcal{X}, \alpha)$ and $g \in G$. By ergodicity $(\mathcal{X}, \alpha)$ must be a singleton.

The purpose of the remaining part of our paper is to prove the converse to Corollary 3.10 and establish a few other conditions equivalent to polynomial growth. The following auxiliary results preceding Theorem 3.16 and their proofs are essentially contained in the work of Azencott [2, pp. 106-111].

Let $\mathfrak{g}$ be a (real) Lie algebra and let $\operatorname{rad}(\mathfrak{g})$ denote the radical. We shall say that $\mathfrak{g}$ is of type $R_{0}$ if for every $X \in \operatorname{rad}(\mathfrak{g})$ the eigenvalues of $\operatorname{ad} X$ are imaginary.

Lemma 3.11. If $\mathfrak{g}$ is of type $R_{0}$ and $\mathfrak{i}$ is a solvable ideal, then $\mathfrak{g} / \mathfrak{i}$ is also of type $R_{0}$.

Proof. We have $\operatorname{rad}(\mathfrak{g} / \mathfrak{i})=\operatorname{rad}(\mathfrak{g}) / \mathfrak{i}$. The lemma can then be proven by an argument analogous to that used in the proof of Lemma 3.5.

Lemma 3.12. Let $\mathfrak{n}$ denote the nilpotent ideal $\mathfrak{n}=[\mathfrak{g}, \operatorname{rad}(\mathfrak{g})]$ of $\mathfrak{g}$ and $\mathfrak{z}$ the centre of $\mathfrak{n}$. Set $\mathfrak{i}=[\mathfrak{n}, \mathfrak{n}] \cap \mathfrak{z}$. It follows that $\mathfrak{g}$ is type $R_{0}$ if and only if $\mathfrak{g} / \mathfrak{i}$ is.

Proof. By the preceding lemma it remains to show that $\mathfrak{g}$ is type $R_{0}$ whenever $\mathfrak{g} / \mathfrak{i}$ is. Let $\pi: \mathfrak{g} \rightarrow \mathfrak{g} / \mathfrak{i}$ be the quotient homomorphism. Denote by $\mathfrak{g}_{\mathbb{C}}$ a complexification of $\mathfrak{g}$ and by $\mathfrak{i}_{\mathbb{C}}, \mathfrak{n}_{\mathbb{C}} \subseteq \mathfrak{g}_{\mathbb{C}}$ the associated complexification of $\mathfrak{i}$ and $\mathfrak{n}$. Then $\mathfrak{g}_{\mathbb{C}} / \mathfrak{i}_{\mathbb{C}}$ is a complexification of $\mathfrak{g} / \mathfrak{i}$ and the quotient map $\pi_{\mathbb{C}}: \mathfrak{g}_{\mathbb{C}} \rightarrow \mathfrak{g}_{\mathbb{C}} / \mathfrak{i}_{\mathbb{C}}$ coincides with the complexification of the quotient map $\pi$. Let $X \in \operatorname{rad}(\mathfrak{g})$ and let $V \in \mathfrak{g}_{\mathbb{C}}$ be an eigenvector of $\operatorname{ad} X$ with equivalue $\lambda$. If $V \notin \mathfrak{i}_{\mathbb{C}}$ then $\pi_{\mathbb{C}} V$ is an eigenvector of $\operatorname{ad}(\pi X)$ with eigenvalue $\lambda$. As $\pi X \in \operatorname{rad}(\mathfrak{g} / \mathfrak{i}), \lambda$ must be imaginary. Thus it remains to consider the case that $V \in \mathfrak{i}_{\mathbb{C}}$.

Let $S$ denote the semisimple part of adX acting on $\mathfrak{g}_{\mathbb{C}}$. As $\mathfrak{i}_{\mathbb{C}}$ and $\mathfrak{n}_{\mathbb{C}}$ are invariant under $S$, there exists a $S$-invariant subspace $\mathfrak{m} \subseteq \mathfrak{n}_{\mathbb{C}}$ such that $\mathfrak{n}_{\mathbb{C}}=\mathfrak{i}_{\mathbb{C}} \oplus \mathfrak{m}$. Note that $V \in \mathfrak{i}_{\mathbb{C}} \subseteq\left[\mathfrak{n}_{\mathbb{C}}, \mathfrak{n}_{\mathbb{C}}\right]=[\mathfrak{m}, \mathfrak{m}]$ because $\mathfrak{i}_{\mathbb{C}}$ is central in $\mathfrak{n}_{\mathbb{C}}$. 
Let $Y_{1}, \ldots, Y_{m}$ be a basis in $\mathfrak{m}$ consisting of eigenvectors of $S$ with eigenvalues $\lambda_{1}, \ldots, \lambda_{m}$. As the linear operator on $\mathfrak{g}_{\mathbb{C}} / \mathfrak{i}_{\mathbb{C}}$ induced by $S$ coincides with the semisimple part of $\operatorname{ad}(\pi X)$, we conclude that $\lambda_{1}, \ldots, \lambda_{m}$ are imaginary. Now, $[\mathfrak{m}, \mathfrak{m}]$ has a basis $\left\{\left[Y_{i}, Y_{j}\right]\right\}_{(i, j) \in I}$ where $I \subseteq\{1,2, \ldots, m\}^{2}$. As the semisimple part of a derivation is a derivation [3, Proposition 4 ; p. 11], $\left[Y_{i}, Y_{j}\right],(i, j) \in$ $I$ are eigenvectors of $S$ with imaginary eigenvalues. But $S V=\lambda V$ and $V \in[\mathfrak{m}, \mathfrak{m}]$. Therefore $\lambda$ must be imaginary.

Corollary 3.13. Let $\mathfrak{n}=[\mathfrak{g}, \operatorname{rad}(\mathfrak{g})]$. It follows that $\mathfrak{g}$ is of type $R_{0}$ if and only if $\mathfrak{g} /[\mathfrak{n}, \mathfrak{n}]$ is.

Proof. $\Rightarrow$ : Lemma 3.11 .

$\Leftarrow$ : When $\mathfrak{n}$ is abelian the claim is trivially true. When $\mathfrak{n}$ is not abelian, it is nilpotent of class $k \geq 2$. Hence, one can find $X \in \mathfrak{n}$ such that $\{0\} \neq$ $[X, \mathfrak{n}] \subseteq \mathfrak{z}$, where $\mathfrak{z}$ is the centre of $\mathfrak{n}$. So $\mathfrak{i}=[\mathfrak{n}, \mathfrak{n}] \cap \mathfrak{z} \neq\{0\}$. Note that $\mathfrak{n} / \mathfrak{i}=$ $[\mathfrak{g} / \mathfrak{i}, \operatorname{rad}(\mathfrak{g} / \mathfrak{i})]$ and the Lie algebra $\mathfrak{g} /[\mathfrak{n}, \mathfrak{n}]$ is isomorphic to $(\mathfrak{g} / \mathfrak{i}) /[\mathfrak{n} / \mathfrak{i}, \mathfrak{n} / \mathfrak{i}]$. Thus we can use Lemma 3.12 and proceed by induction in the dimension of $\mathfrak{g}$.

Lemma 3.14. Let $G$ be a connected Lie group with Lie algebra $\mathfrak{g}$ not of type $R_{0}$. Then there exists a $G$-space $\mathcal{X}$ such that :

(1) $\mathcal{X}$ is a finite dimensional vector space,

(2) the function $G \times \mathcal{X} \ni(g, x) \rightarrow g x$ is (real) analytic,

(3) for every compact $K \subseteq \mathcal{X}$ and every neighbourhood $U$ of 0 in $\mathcal{X}$ there exists $g \in G$ with $g K \subseteq U$,

(4) there exists $g \in G$ and $v \in \mathcal{X}-\{0\}$ such that $g x=x+v$ for all $x \in \mathcal{X}$.

Proof. Let $\mathfrak{n}=[\mathfrak{g}, \operatorname{rad}(\mathfrak{g})], \tilde{\mathfrak{g}}=\mathfrak{g} /[\mathfrak{n}, \mathfrak{n}], \widetilde{\mathfrak{n}}=\mathfrak{n} /[\mathfrak{n}, \mathfrak{n}]=[\tilde{\mathfrak{g}}, \operatorname{rad}(\widetilde{\mathfrak{g}})]$. Denote by $\tilde{\mathfrak{g}}_{\mathbb{C}}$ a complexification of $\widetilde{\mathfrak{g}}$ and by $\widetilde{\mathfrak{n}}_{\mathbb{C}} \subseteq \widetilde{\mathfrak{g}}_{\mathbb{C}}$ the associated complexification of $\tilde{\mathfrak{n}}$. Let $\rho(\tilde{X}), \tilde{X} \in \widetilde{\mathfrak{g}}$ denote ad $\widetilde{X}$ acting on $\tilde{\mathfrak{n}}_{\mathbb{C}}$. As $\tilde{\mathfrak{n}}$ is abelian, $\rho(\tilde{\mathfrak{n}})=\{0\}$ and due to the definition of $\tilde{\mathfrak{n}}$ we have $[\rho(\tilde{\mathfrak{g}}), \rho(\operatorname{rad}(\tilde{\mathfrak{g}}))]=\{0\}$. Let $S$ be the complex subspace of $\operatorname{End}\left(\widetilde{\mathfrak{n}}_{\mathbb{C}}\right)$ spanned by $\rho(\operatorname{rad}(\tilde{\mathfrak{g}}))$. Then $S$ is a commuting family of operators. Let $n$ be the dimension of $\tilde{\mathfrak{n}}_{\mathbb{C}}$. By [3, Théorème 1 ; p. 10 and Proposition 5 ; p. 12] there exist linear functionals $\alpha_{1}, \ldots, \alpha_{k}: S \rightarrow \mathbb{C}$ such that for every $i=1,2, \ldots, k \tilde{\mathfrak{n}}_{i}=\bigcap_{A \in S} \operatorname{Ker}(A-$ $\left.\alpha_{i}(A)\right)^{n} \neq\{0\}$ and $\widetilde{\mathfrak{n}}_{\mathbb{C}}=\widetilde{\mathfrak{n}}_{1} \oplus \cdots \oplus \widetilde{\mathfrak{n}}_{k}$. Since $[\rho(\widetilde{\mathfrak{g}}), \rho(\operatorname{rad}(\tilde{\mathfrak{g}}))]=\{0\}$, each $\widetilde{\mathfrak{n}}_{i}$ is invariant under $\rho(\tilde{\mathfrak{g}})$.

From Lemma 3.13 we know that $\tilde{\mathfrak{g}}$ is not type $R_{0}$, so there exists $\tilde{X}_{0} \in$ $\operatorname{rad}(\tilde{\mathfrak{g}})$ such that $\operatorname{ad} \widetilde{X}_{0}$ has an eigenvalue $\lambda$ with $\operatorname{Re} \lambda \neq 0$. Note that if $\widetilde{V} \in \widetilde{\mathfrak{g}}_{\mathbb{C}}$ and $\left(\operatorname{ad} \widetilde{X}_{0}\right) \tilde{V}=\lambda \widetilde{V}$, then $\tilde{V} \in \widetilde{\mathfrak{n}}_{\mathbb{C}}$. Therefore $\lambda$ is an eigenvalue of $\rho\left(\widetilde{X}_{0}\right)$ and we can assume that $\widetilde{V} \in \tilde{\mathfrak{n}}_{1}$. Then $\lambda=\alpha_{1}\left(\rho\left(\tilde{X}_{0}\right)\right)$. Note also that $\widetilde{X}_{0} \notin \widetilde{\mathfrak{n}}$.

Let $\tilde{\mathfrak{n}}_{0}$ be the subspace of $\tilde{\mathfrak{n}}_{1}$ spanned by $\bigcup_{A \in S}\left(A-\alpha_{i}(A)\right) \tilde{\mathfrak{n}}_{1}$. As $S_{1}=$ $\left\{A \uparrow \tilde{\mathfrak{n}}_{1} ; A \in S\right\}$ is a (complex) solvable Lie subalgebra of End $\left(\widetilde{\mathfrak{n}}_{1}\right)$, by Lie's 
theorem there exists a basis $e_{1}, \ldots, e_{l}$ in $\tilde{\mathfrak{n}}_{1}$ such that the matrix of each $A \in$ $S_{1}$ is upper triangular with respect to this basis. Since $\left(A-\alpha_{1}(A)\right)^{n}\left\lceil\widetilde{\mathfrak{n}}_{1}=0\right.$ for every $A \in S$, we conclude that $e_{l} \notin \tilde{\mathfrak{n}}_{0}$. So $\tilde{\mathfrak{n}}_{0} \neq \tilde{\mathfrak{n}}_{1}$ and, consequently, $\widetilde{\mathfrak{m}}=\widetilde{\mathfrak{n}}_{0} \oplus \tilde{\mathfrak{n}}_{2} \oplus \cdots \oplus \tilde{\mathfrak{n}}_{k} \neq \widetilde{\mathfrak{n}}_{\mathbb{C}}$. Note that $\widetilde{\mathfrak{m}}$ is invariant under $\rho(\widetilde{\mathfrak{g}})$. Furthermore, $\rho\left(\tilde{X}_{0}\right) \tilde{X}-\lambda \tilde{X} \in \tilde{\mathfrak{m}}$ for every $\tilde{X} \in \tilde{\mathfrak{n}}_{\mathbb{C}}$.

Next define $\widetilde{\mathfrak{l}}=\mathbb{C} \widetilde{X}_{0}+\widetilde{\mathfrak{n}}_{\mathbb{C}}$. Due to the definition of $\tilde{\mathfrak{n}}, \tilde{\mathfrak{l}}$ is invariant under $\operatorname{ad} \tilde{X}, \tilde{X} \in \tilde{\mathfrak{g}}$. We shall write $\rho^{\prime}(\tilde{X}), \tilde{X} \in \tilde{\mathfrak{g}}$ for ad $\tilde{X}$ acting on $\tilde{\mathfrak{l}}$. We now have subspaces $\widetilde{\mathfrak{m}} \subseteq \widetilde{\mathfrak{n}}_{\mathbb{C}} \subseteq \tilde{\mathfrak{l}}, \tilde{\mathfrak{m}} \neq \widetilde{\mathfrak{n}}_{\mathbb{C}} \neq \widetilde{\mathfrak{l}}$, invariant under $\rho^{\prime}(\tilde{\mathfrak{g}})$. We set $\mathcal{L}=\tilde{\mathfrak{l}} / \tilde{\mathfrak{m}}, \mathcal{X}=\tilde{\mathfrak{n}} / \tilde{\mathfrak{m}}$ and write $\rho^{\prime \prime}(\widetilde{X})$ for $\rho^{\prime}(\tilde{X})$ acting on $\mathcal{L}$. Furthermore, we write $p: \widetilde{\mathfrak{l}} \rightarrow \mathcal{L}$ for the quotient map. It is straightforward to check that $\rho^{\prime \prime}\left(\tilde{X}_{0}\right) x=\lambda x$ for all $x \in \mathcal{X}$, and $\rho^{\prime \prime}(\widetilde{X})\left(x+p\left(\tilde{X}_{0}\right)\right)=-\lambda p(\tilde{X})$ for $\tilde{X} \in \tilde{\mathfrak{n}}$ and $x \in \mathcal{X}$. Moreover, $\rho^{\prime \prime}(\widetilde{\mathfrak{g}}) \mathcal{L} \subseteq \mathcal{X}$, and $\rho^{\prime \prime}(\widetilde{X})^{2}=0$ for $\tilde{X} \in \widetilde{\mathfrak{n}}$ (since $\tilde{\mathfrak{n}}$ is abelian).

We now introduce a linear representation $\varphi$ of $G$ on $\mathcal{L}$ as follows. We start with the adjoint representation of $G$ on $\mathfrak{g}$. This defines canonically the quotient representation on $\tilde{\mathfrak{g}}=\mathfrak{g} /[\mathfrak{n}, \mathfrak{n}]$ and also a representation $\psi$ on $\mathfrak{g}_{\mathbb{C}}$. It is clear that $\psi\left(e^{X}\right)=e^{\operatorname{ad}(\pi X)}, X \in \mathfrak{g}$, where $\pi: \mathfrak{g} \rightarrow \tilde{\mathfrak{g}}$ the quotient homomorphism. Therefore $\tilde{\mathfrak{l}}, \mathfrak{n}_{\mathbb{C}}$, and $\mathfrak{m}$ are invariant under $\psi$. We define $\varphi$ as the quotient by $\widetilde{\mathfrak{m}}$ of the subrepresentation of $\psi$ on $\widetilde{\mathfrak{l}}$. Then we have $\varphi\left(e^{X}\right)=e^{\rho^{\prime \prime}(\pi(X))}, \mathcal{X}$ is invariant under $\varphi$, and $\varphi(g) p\left(\widetilde{X}_{0}\right)-p\left(\widetilde{X}_{0}\right) \in \mathcal{X}$ for all $g \in G$. One then checks that the formula $g x=\varphi(g)\left(x+p\left(\widetilde{X}_{0}\right)\right)-p\left(\tilde{X}_{0}\right)$ defines a $G$-action on $\mathcal{X}$. If $\mathcal{X}$ is considered as a real vector space, this $G$-action satisfies our requirement (2) (because the adjoint representation is analytic, and taking subrepresentation and quotients preserves analycity). Let us now see that (3) is also satisfied. Indeed, let $K \subseteq \mathcal{X}$ be compact and $U \subseteq \mathcal{X}$ be a neighbourhood of 0 . Let $\|\cdot\|$ be any norm on $V$. Then $K$ is contained in a ball $B(0, R)=\{x \in V ;\|x\|<R\}$ while $U$ contains a ball $B(0, r)$. Recall that $\rho^{\prime \prime}\left(\widetilde{X}_{0}\right) x=\lambda x$ for all $x \in \mathcal{X}$, where $\operatorname{Re} \lambda \neq 0$. Moreover, $\rho^{\prime \prime}\left(\widetilde{X}_{0}\right) p\left(\widetilde{X}_{0}\right)=0$. We can, of course, assume that $\operatorname{Re} \lambda<0$. As $\widetilde{X}_{0}=\pi\left(X_{0}\right)$ for some $X_{0} \in \mathfrak{g}$, we have $e^{X_{0}} x=e^{\rho^{\prime \prime}\left(\widetilde{X}_{0}\right)} x=e^{\lambda} x$. It is then clear that we can satisfy (3). Finally, when $X \in \mathfrak{n}$, then $e^{X} x=e^{\rho^{\prime \prime}(\pi(X))} x=x-\lambda p(\pi(X))$. Since $p(\pi(\mathfrak{n})) \neq\{0\},(4)$ is also satisfied.

Corollary 3.15. Let $G$ be a connected Lie group whose Lie algebra $\mathfrak{g}$ is not of type $R_{0}$. Then there exists an absolutely continuous compactly supported probability measure on $G$ admitting a nontrivial bounded harmonic function.

Proof. With the notation of Lemma 3.14, let $\|\cdot\|$ be any norm on $\mathcal{X}$. Set $U=$ $\{x \in \mathcal{X} ;\|x\|<1\}, K=\{x \in \mathcal{X} ;\|x\| \leq 1\}$, and $S=\{g \in G ; g K \subseteq U\}$. By Lemma $3.14, S$ is a nonempty open semigroup in $G$. Let $\mu$ be any compactly supported absolutely continuous probability measure with $\mu(S)=1$.

Let $\delta_{0}$ be the point measure concentrated in $0 \in \mathcal{X}$, and set $\rho_{n}=\frac{1}{n} \sum_{i=1}^{n} \mu^{i} *$ 
$\delta_{0}$. It is clear that $\rho_{n}(K)=1$ for all $n$. Thus using Prohorov's theorem [8, Theorem 1.1.11], $\left\{\rho_{n}\right\}_{n=1}^{\infty}$ is a weakly relatively compact set of probability measures. If $\rho$ is its cluster point then $\rho(K)=1$ and $\mu * \rho=\rho$. Let $f: \mathcal{X} \rightarrow[0,1]$ be a continuous function of compact support such that $f(K)=\{1\}$. Then $h(g)=\int f(g x) \rho(d x)$ is a bounded $\mu$-harmonic function. Using (4) of Lemma 3.14 and the fact that $\rho$ has compact support we can see that $h$ is not constant.

Theorem 3.16. For a connected lcsc group $G$ the following conditions are equivalent:

(a) $G$ has polynomial growth,

(b) every SAT $G$-space is singleton,

(c) every SAT homogeneous space of $G$ is a singleton,

(d) every homogeneous space of $G$ admits a $\sigma$-finite invariant measure,

(e) the Choquet-Deny theorem holds for every spread out probability measure on $G$,

(f) the Choquet-Deny theorem holds for every compactly supported absolutely continuous probability measure on $G$.

Proof. The implication $(\mathrm{a}) \Rightarrow(\mathrm{b})$ is contained in Corollary $3.10,(\mathrm{~b}) \Rightarrow(\mathrm{e})$ follows from Lemma 2.3 , and $(\mathrm{e}) \Rightarrow(\mathrm{f})$ is trivial. To prove that (f) implies (a) suppose that (f) is true but (a) is false. If $G$ is not amenable then every spread out probability measure on $G$ has nontrivial $\mu$-boundary [5, p. 213], [23, Proposition 1.9] and we obtain a contradiction. Assume that $G$ is amenable. Let $K \subseteq G$ be a compact normal subgroup such that $\widetilde{G}=G / K$ is a Lie group. Since $G$ is not of polynomial growth, $\widetilde{G}$ is also not of polynomial growth [7, Théorème 1.4]. But $\widetilde{G}$ is amenable so $\widetilde{G} / \operatorname{rad}(\widetilde{G})$ is compact $[\mathbf{1 9}$, Theorem 3.8]. Therefore $\operatorname{rad}(\widetilde{G})$ is not of polynomial growth [7, Théorème 1.4]. Hence, the Lie algebra of $\operatorname{rad}(\widetilde{G})$ is not type $R$ [13, Theorem 1.4] and, consequently, the Lie algebra of $\widetilde{G}$ is not type $R_{0}$. Thus by Corollary 3.15 there exists a compactly supported absolutely continuous probability measure on $\widetilde{G}$ with nontrivial $\widetilde{\mu}$-boundary. As $K$ is compact we can find a compactly supported absolutely continuous probability measure $\mu$ on $G$ such that $\widetilde{\mu}=\pi \mu$, where $\pi: G \rightarrow \widetilde{G}$ is the canonical homomorphism. It is easy to see that the $\mu$-boundary cannot be trivial. We again arrive at a contradiction. We have thus established the equivalence $(\mathrm{a}) \Leftrightarrow(\mathrm{b}) \Leftrightarrow(\mathrm{e}) \Leftrightarrow(\mathrm{f})$.

To prove that (a) implies (d) we note that every closed subgroup of a group of polynomial growth has polynomial growth and polynomial growth implies unimodularity [7, Théorème 1.2 and Lemme 1.3]. Combining this with the well-known condition for existence of invariant measures on homogeneous spaces [25, Theorem $8.30 ;$ p. 40] we obtain (d). Next (d) implies (c) because 
every SAT action preserving a $\sigma$-finite invariant measure is necessarily purely atomic [9, Proposition 2.6] and we can then repeat the proof of Corollary 3.10. To complete our proof it suffices to show that (c) implies (f). This can be achieved by applying a special case of the following result of Raugi [21, Théorème 13.4]: If $G$ is lcsc, $G / G_{0}$ is compact, and $\mu$ is a spread out probability measure on $G$ with finite first order moment, then the $\mu$ boundary can be realized as a homogeneous space of $G$. Since compact support implies finite first order moment, (c) combined with Lemma 2.3 implies (f).

Remark 3.17. In [9] we proved that a finitely generated solvable group $G$ has polynomial growth if and only if it does not admit nonatomic SAT actions. The proof was based on Rosenblatt's theorem [22] stating that $G$ has polynomial growth if and only if it does not contain a free subsemigroup on two generators. Using the fact that free semigroups are nonamenable we exhibited a random walk on $G$ whose $\mu$-boundary was nonatomic. For solvable connected lcsc the proof of the implication $(\mathrm{f}) \Rightarrow(\mathrm{a})$ in Theorem 3.16 can be obtained by a completely analogous argument relying on the fact that such $G$ has polynomial growth if and only if every open subsemigroup of $G$ is amenable [12, Theorem 3], [19, Theorem 6.39]. This result was originally formulated by Jenkins [12] for arbitrary connected $G$, however, his proof seems complete only in the solvable case. The auxiliary argument summarized in Corollary 3.15 enabled us to prove (f) $\Rightarrow$ (a) without involving Jenkins' theorem.

Acknowledgement: The author wishes to thank David Handelman for his support at the University of Ottawa where this work was carried out.

Note added in proof: A complete proof of the Jenkins' claim mentioned in Remark 3.17 can be found in the author's Exponential boundedness and amenability of open subsemigroups of locally compact groups, Canadian J. Math., 46 (1994), 1263-1274.

\section{References}

[1] A. Avez, Harmonic functions on groups, In: Cahen and Flato (eds.): Differential geometry and Relativity, Reidel, Dordrecht 1976, 27-32.

[2] R. Azencott, Espaces de Poisson des groupes localement compacts, Lecture Notes in Mathematics., vol 148, Springer, Berlin 1970.

[3] N. Bourbaki, Éléments de mathématique. Groupes et algèbres de Lie, Chaps. VII, VIII, Hermann, Paris 1975.

[4] E.G. Effros, Transformation groups and $C^{*}$-algebras, Annals of Math., 81 (1965), 38-55. 
[5] H. Furstenberg, Boundary theory and stochastic processes on homogeneous spaces, Proc. Sympos. Pure Math., vol 26: Harmonic analysis on homogeneous spaces, AMS, Providence, R.I. 1973, 193-229.

[6] M. Gromov, Groups of polynomial growth and expanding maps, Publ. Math. I.H.E.S., 53 (1981), 53-73.

[7] Y. Guivarc'h, Croissance polynomiale et periodes des fonctions harmoni-ques, Bull. Soc. Math. France, 101 (1973), 333-379.

[8] H. Heyer, Probability measures on locally compact groups, Springer, Berlin 1977.

[9] W. Jaworski, Strongly approximately transitive group actions, the Choquet-Deny theorem, and polynomial growth, Pacific J. Math., 165 (1994), 115-129.

[10] , unpublished manuscript.

[11] - Poisson and Furstenberg boundaries of random walks, C.R. Math. Rep. Acad. Sci. Canada, XIII (1991), 279-284.

[12] J.W. Jenkins, Folner's condition for exponentially bounded groups, Math. Scand., 35 (1974), 165-174.

[13] , Growth of connected locally compact groups, J. Funct. Analysis, 12 (1973), 113-127.

[14] M.I. Kargapolov and Ju.I. Merzljakov, Fundamentals of the theory of groups, Springer, New York 1979.

[15] V. Losert, On the structure of groups with polynomial growth, Math. Z., 195 (1987), 195-217.

[16] G.W. Mackey, Point realizations of transformation groups, Illinois J. Math., 6 (1962), 327-335.

[17] J. Neveu, Mathematical foundations of the calculus of probability, Holden-Day, San Francisco 1965.

[18] O.A. Nielsen, Direct integral theory, Lecture notes in pure and applied math., vol. 61, Marcel Dekker, New York 1980.

[19] A.L.T. Paterson, Amenability, AMS, Providence 1988.

[20] D. Revuz, Markov chains, North-Holland, Amsterdam 1975.

[21] A. Raugi, Fonctions harmoniques sur les groupes localement compacts a base denombrable, Bull. Soc. Math. France, Mémoire, 54 (1977), 5-118.

[22] J.M. Rosenblatt, Invariant measures and growth conditions, Trans. Amer. Math. Soc., 193 (1974), 33-35.

[23] - Ergodic and mixing random walks on locally compact groups, Math. Ann., 257 (1981), 31-42.

[24] V.S. Varadarajan, Groups of automorphisms of Borel spaces, Trans. Amer. Math. Soc., 109 (1963), 191-220.

[25] Geometry of quantum theory, Vol. 2, Van Nostrand 1970.

[26] R.J. Zimmer, Amenable ergodic groups actions and an application to Poisson boundaries of random walks, J. Funct. Analysis, 27 (1978), 350-372.

[27] - Ergodic theory and semisimple groups, Birkhauser, Boston 1984.

Received January 12, 1993. 



\section{CONTENTS}

N. Ben Amar, Tangential deformations on the dual of nilpotent special Lie

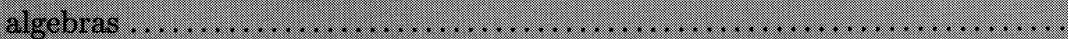

Martin Bendersky, Donald M. Davis and Mark Mahowald, $v_{1}$-periodic

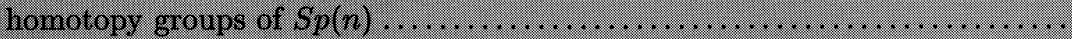

Georgia Benkart, Seok-Jin Kang, Kailash C. Misra, Indefinite Kac-

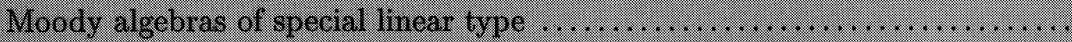

Robin Brooks and Charles Odenthal, Nielsen numbers for roots of maps

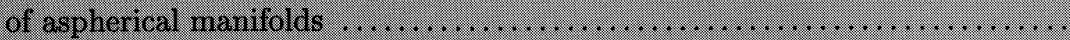

L.J. Bunce and J.D. Maitland Wright, Velocity maps in von Neumann

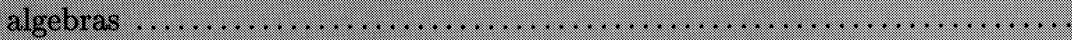

Bradley N. Currey, Smooth decomposition of finite multiplicity monomial representations for a class of completely solvable homogeneous spaces ...... R.J. Daverman and D.F. Snyder, On proper surjections with locally triv-

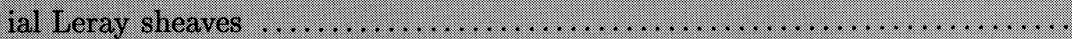

Patrick N. Dowling, Zhibao Hu and Mark A. Smith, MLUR renormings

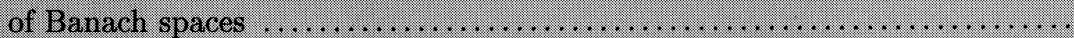
Tuval Foguel, Finite groups with a special 2-generator property ..........

Mourad E.H. Ismail and Mizan Rahman, Some basic bilateral sums and

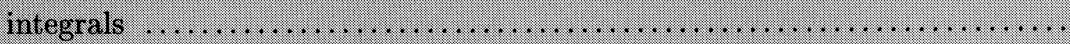

Wojciech Jaworski, Strong approximate transitivity, polynomial growth, and spread out random walks on locally compact groups $\ldots \ldots \ldots \ldots \ldots . . . . . .$.

N. Kutev and F. Tomi, Nonexistence and instability in the exterior Dirichlet problem for the minimal surface equation in the plane............

A. Nobile, Equisingularity Theory for Plane Curves With Embedded

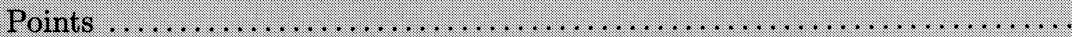

Dominikus Noll, Directional differentiability of the metric projection in Hilbert space 


\section{PACIFIC JOURNAL OF MATHEMATICS}

Volume $170 \quad$ No. $2 \quad$ October 1995

Tangential deformations on the dual of nilpotent special Lie algebras

297

NABIHA BEN AMAR

$v_{1}$-periodic homotopy groups of $S p(n)$

319

MaRTIN BENDERSKY, DONALD M. DAVIS and MARK MAHOWALD

Indefinite Kac-Moody algebras of special linear type

GeOrgia BenKart, SEOK-Jin KANG and KaILASh C. MisRa

Nielsen numbers for roots of maps of aspherical manifolds

405

ROBIN B. S. BROOKS and CHARLES ODENTHAL

Velocity maps in von Neumann algebras

L. J. BUNCE and JOHN DAVID MAITLAND WRIGHT

Smooth decomposition of finite multiplicity monomial representations for a class of 429 completely solvable homogeneous spaces

\section{BRADLEY CURREY}

On proper surjections with locally trivial Leray sheaves

ROBERT JAY DAVERMAN and DAVID FRED SNYDER

MLUR renormings of Banach spaces

PATRICK DOWLING, Zhibao Hu and MARK ANDREW SMIth

Finite groups with a special 2-generator property

TUVAL S. Foguel

Some basic bilateral sums and integrals

MOURAD ISMAIL and MIZAN RAHMAN

Strong approximate transitivity, polynomial growth, and spread out random walks on 517 locally compact groups

WOJCIECH JAWORSKI

Nonexistence and instability in the exterior Dirichlet problem for the minimal surface 535 equation in the plane

NiKolai KuteV and FriedRich TOMI

Equisingularity theory for plane curves with embedded points

Augusto Nobile

Directional differentiability of the metric projection in Hilbert space

DOMINIKUS NOLL 